\title{
What do asthmatic patients think about telemedicine visits?
}

\author{
${ }^{1}$ Allergy Centre, CUF Descobertas Hospital, Lisbon, Portugal \\ ${ }^{2}$ Department of Pulmonology, Central Hospital of Funchal, Funchal, Portugal \\ ${ }^{3}$ Department of Pulmonology, Hospital Centre of Barreiro-Montijo, Barreiro, Portugal \\ ${ }^{4}$ Portuguese Association of Asthmatics, Porto, Portugal
}

\section{KEY WORDS}

Asthma; COVID-19; survey;

telemedicine; virtual visits.

\section{Corresponding author}

Cláudia Sousa

Department of Pulmonology

Hospital Central do Funchal

Av. Luís de Camões 6180

9000-177 Funchal, Portugal

E-mail: claudiasabinafsousa@gmail.com

Doi

10.23822/EurAnnACI.1764-1489.182

\begin{abstract}
Summary
Introduction. Due to the Coronavirus disease 2019 (COVID-19) outbreak and the national emergency state, virtual visits were implemented as an alternative to in-person visits. With this study we aimed to establish asthma patients' general satisfaction with the quality of health care provided by virtual visits (phone or video calls). Materials and methods. A questionnaire (9 questions) was published on the Facebook page of the Portuguese Association of Asthmatics. It was available online for general self-reported asthmatic patients to answer during one month, starting on $11^{\text {st }}$ May 2020. The survey only allowed one answer per registered user. Results. Fifty-five responses were obtained. Patients were satisfied with communication with providers (> 88\%); nevertheless, one-half evaluated the virtual visit as inferior when compared to in-person visits. About one third attributed a classification of 6 or less $(0-10$ scale, 0 being the worst and 10 the best consultation possible), but still most of the patients would either recommend it or use this kind of medical visits in the future, even outside the actual pandemic context. Patients also referred some important limitations, as lack of physical examination and the fact that the medical visit was more impersonal. Only 27\% had technical issues accessing virtual visits. Positive aspects were also named, such as virtual visits being practical and avoiding the need to move to the hospital. Discussion and conclusions. Our survey revealed that small changes could further increase patients' satisfaction, adherence and confidence in telemedicine. Although presenting some limitations, virtual visits seem to be generally well accepted by asthmatic patients and it might be a good alternative for in-person visits, at least in such difficult times when social distancing is recommended.
\end{abstract}

\section{Introduction}

Worldwide coronavirus disease 2019 (COVID-19) brought a lot of challenges to healthcare organizations, including safety measures, with the need to restrict the number of face-to-face visits (1). Telemedicine is capable to overcome the distance and safety barriers in this context and might be as effective as in-person visits for outpatient management of asthma (2), enabling mild to moderate-severe patients to get the supportive care they need. Several authors documented that virtual visits (VV), that could be either video or phone calls, for asthma patients allow positive outcomes, such as more symptom-free days and fewer emergency department visits or hospitalizations, improving asthma control $(3,4)$. Moreover, it was demonstrated that VV are comparable to in-person visits, enabling its occasional replacement with same outcomes in asthma control (5).

Every patient might be at risk of SARS-CoV-2 exposure (6) and to reduce such risk, as it successfully occurred in many other medical specialties around the globe (7-11), allergy centers implemented VV as an alternative to in-person visits (12). As telemedicine programs were nationally applied, we became curious about the acceptability and satisfaction of asthmatic patients with this type of virtual visits. With this study we aimed to establish self-reported asthma patients' general satisfaction with the quality of health care provided in VV during the recent $\mathrm{Na}$ tional Emergency State in Portugal. 


\section{Materials and methods}

The authors conducted an online survey consisting of eight multiple-choice questions and one optional open-ended question for asthma patients that had recently participated in VV (either phone or video calls), partially adapted from the questionnaire used by Donelan K. et al. (13). The survey addressed three main domains of virtual visits: communication with the provider, quality of the visit and technical difficulties in online access. Quality of the visit was accessed asking the patient to rate it in a scale from 0 to 10,0 being the worst and 10 the best consultation possible and asking them to compare to an in-person visit. The online survey only enabled the same user to answer once, except if using another computer/e-mail. The survey was published on the Portuguese Association of Asthmatics Facebook page, being available online for one month starting on $11^{\text {th }}$ May 2020. Patients were self-reported asthmatic patients having access to this Facebook page. The questionnaire was specifically addressed to self-reported asthma patients and no information regarding personal characteristics was asked, enabling a shorter survey, and overcoming potential privacy issues.

\section{Results}

We obtained 55 replies to our survey. The survey (freely translated to English language) and respective answers are shown in table I. Patients were satisfied with communication with the provider (87,5\% said the clinician listened carefully to their questions or complaints, and $90,9 \%$ said the clinician exposed things clearly). The length of the appointment was adequate for $76,3 \%$ as they were satisfied with the amount of time the doctor spent with them. One-half of the patients evaluated the teleconsultation as inferior when compared to in-person visits and about one third attributed a classification of 6 or less to it. Only $27,3 \%$ had some technical issues accessing the virtual visit and the majority of patients would either recommend it or use this model of visits in the future, even outside actual pandemic context.

In addition to the answers to the pre-established questions, patients were given the possibility to point out some critics or compliments in the last question (optional and open-ended). Twenty-seven patients answered to this optional question (table II). In this open-ended question patients signaled as negative aspects the lack of physical examination and the fact that the medical visit was more impersonal. Compliments were given to the fact that it was a very practical and fast way to access a medical appointment and avoided to move by transportations to the hospital.

\section{Discussion}

As it was also previously found by other authors outside this pandemic context (13), patients reported an overall satisfaction with VV during the COVID-19 outbreak. Communication between patients and providers was not compromised in this model of appointments (> 85\% were satisfied with both explanation and active listening by the doctor). Other reports documented similar results, as it was found in a systematic review of 32 studies suggesting that $\mathrm{VV}$ were acceptable to patients in several circumstances (14).

Nevertheless, to obtain an increase of VV in daily practice much can be learned from this survey, and some aspects have to be improved in the future. Furthermore, the pandemic context might interfere with patient's expectations and lead to a perception of an overall satisfaction that otherwise would not be noted. About one-half of patients ranked their last $\mathrm{VV}$ as inferior to in-person visits and one-third of them attributed a classification of 6 or less to these appointments. Complementing this information with limitations pointed out in the open answer question, the major concern for the patients was the lack of physical examination, so it can be hypothesized that this is the main factor preventing further acceptance to this telemedicine tool. Some smartphone apps have been tested for the detection and analysis of both cardiac and pulmonary auscultation sounds, and might constitute a future solution to overcome this limitation of virtual visits $(15,16)$. Although the identified limitations, most patients would recommend VV to their friends and family members and would use it in the future. This reveals that small changes could further increase patients' satisfaction, adherence and confidence in telemedicine for healthcare assistance.

In addition, patients acknowledge that this kind of appointments is a valuable tool for disease follow-ups and prescription renewal. These results are inconsistent with those found by $\mathrm{Du}-$ plaga M. et al. (17) that stated, patients suffering from chronic respiratory diseases have a high acceptance of e-health applications (appointment booking, prescription renewal, and access to laboratory test results and educational resources) but do not recognize telemedicine as a valuable solution directly related to medical care (communication with healthcare providers and disease monitoring) (17).

Surprisingly, technical issues were a minor difficulty, with only one quarter of the patients reporting technical problems accessing to the VV. Other potential patients' concerns, such as legal, safety or privacy issues (18) were not contemplated in our questionnaire, but remained unreported in the open-ended question. The authors believe that the aspects pointed out by asthma patients are excellent opportunities to improve adherence to VV by asthma patients in the near future. For instance, doctors might clarify patients that in follow-up visits a good clinical history and attention to some physical signals visible by video might partially replace physical examination, despite not being able to perform an important observation step that is pulmonary and cardiac auscultation. This could surpass patients' fears and insecurities that their illness might not be well managed without physical examination, promoting more recognition of the potential of $\mathrm{VV}$. 
Table I - Answers of patients with asthma that participated in virtual medical appointments during the Emergency State.

\section{PATIENTS VIRTUAL VISITS SURVEY $(\mathbf{n}=55)$}

The clinician exposed things clearly or in an easy way for me to understand?

$\left.\begin{array}{l}\text { No } \\ \text { Yes }\end{array}\right] \quad 9.1 \%$

The clinician listened carefully to my complains and questions?

I don't know how to answer

No $-9.1 \%$

Yes

$87.3 \%$

Am I satisfied with the amount of time the doctor spent with me on this visit?

I don't know how to answer $]-5.5 \%$

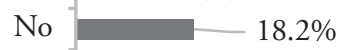

Yes $76.3 \%$

In comparison to the quality of a face- to-face visit, this virtual consultation was:

I don't know how to answer $\quad 5.5 \%$

Superior

Inferior

The same

$50.9 \%$

$38.2 \%$

Rate your virtual visit in a scale from 0 to 10,0 meaning it was the worst medical visit and 10 the best possible medical visit:

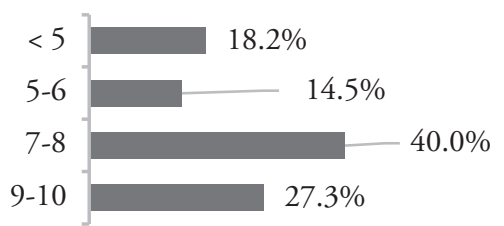

Did you feel any difficulties accessing to this virtual medical visit?



Would you recommend this model of medical visit to your friends and family?

I don't know how to answer $]-0.0 \%$

No $-23.6 \%$

Yes $76.4 \%$

In the future, even if actual contingencies change, will you adhere to this model of appointments if it continues to be available?

I don't know how to answer $]-3.6 \%$

No

Yes

$61.8 \%$ 
Table II - Main critics and compliments pointed out in the open question and the number of patients referring each aspect (one patient may have contributed with more than one critic and/or compliment).

\begin{tabular}{ll}
\hline Some critics and compliments to VIRTUAL VISITS mentioned in open QUESTION \\
\hline COMPLIMENTS & CRITICS \\
\hline Practical and fast $(\mathbf{n}=\mathbf{6})$ & $\begin{array}{l}\text { Only applies when the reason for the appointment does not include } \\
\text { observation }(\mathrm{n}=8) .\end{array}$ \\
Good for prescription renewal $(\mathbf{n}=\mathbf{1})$ & $\begin{array}{l}\text { Very impersonal appointment, doctors seem to follow a pre-defined } \\
\text { protocol: they are limited to asking questions and the user is limited } \\
\text { to just answering }(\mathrm{n}=2) . \\
\text { Sometimes the internet connection fails (video failures, sound or } \\
\text { problems with the delay) }(\mathrm{n}=1) .\end{array}$ \\
Avoids commuting to the hospital $(\mathbf{n}=7)$ & \\
$\begin{array}{l}\text { Good for follow-up appointments }(\mathbf{n}=\mathbf{3}) \\
\text { It allows us not to be helpless in times when face-to-face } \\
\text { appointment would be impractical }(\mathbf{n}=\mathbf{1})\end{array}$ &
\end{tabular}

On the other hand, the creation of a simplified and prioritized way to the doctor arrange a face-to-face medical visit for an indispensable physical examination/treatment discussion after a VV could be a win-win alternative to address this matter.

\section{Limitations}

One limitation is that this survey was applied to any patient with self-reported asthma that were observed in a VV during or before the Emergency weeks in Portugal. There was no discrimination between VV (real-time audio or video). Real-time video-appointments might enable a more empathic relation between patients and their doctors and provide visual signs that could complement physical examination. It would be interesting to investigate whether there are differences in patient satisfaction between these two different telemedicine tools. Although being in line with the literature (14), the size of our sample is another limitation. Despite being available for four weeks, only 55 patient's answers were retrieved, limiting the generalization of results. Another particularity is that the survey didn't include questions addressing patient's general characteristics, such as genre or age, and patients with access to Facebook usually are younger and technology-friendly, which can explain the low rate of technical difficulties accessing VV. This can influence the perception of acceptance and thus might not be extrapolated to the general population or even to other chronic respiratory diseases. As COVID-19 might interfere with patients' expectations, we cannot extrapolate that similar results could be achieved in a post-pandemic period.

\section{Conclusions}

As far as we know, this is the first survey applied to patients in our country regarding telemedicine performed during the COVID-19 pandemic. The results suggest that telemedicine is quickly becom- ing a key add-on to healthcare and might be a good alternative for in-person visits for asthmatic patients, patients, at least in such difficult times when social distancing is recommended, as patients express an overall satisfaction with this type of medical consultations.

\section{Acknowledgments}

Authors appreciate Portuguese Association of Asthmatics' for sharing the online questionnaire.

\section{Conflict of interests}

The authors declare that they have no conflict of interests.

\section{References}

1. Shaker MS, Oppenheimer J, Grayson M, et al. COVID-19: Pandemic Contingency Planning for the Allergy and Immunology Clinic. J Allergy Clin Immunol Pract 2020;8(5):1477-88.e5.

2. Portnoy JM, Pandya A, Waller M, Elliott T. Telemedicine and emerging technologies for health care in allergy/immunology. J Allergy Clin Immunol 2020;145(2):445-54.

3. Halterman JS, Tajon R, Tremblay P, et al. Development of School-Based Asthma Management Programs in Rochester, New York: Presented in Honor of Dr Robert Haggerty. Acad Pediatr 2017;17(6):595-9.

4. Estrada RD, Ownby DR. Rural Asthma: Current Understanding of Prevalence, Patterns, and Interventions for Children and Adolescents. Curr Allergy Asthma Rep 2017;17(6):37.

5. van den Wijngaart LS, Roukema J, Boehmer ALM, et al. A virtual asthma clinic for children: fewer routine outpatient visits, same asthma control. Eur Respir J 2017;50(4).

6. Pfaar O, Klimek L, Jutel M, et al. COVID-19 pandemic: Practical considerations on the organization of an allergy clinic - an EAACI/ ARIA Position. Allergy 2021;76(3):648-76. 
7. Aziz A, Zork N, Aubey JJ, et al. Telehealth for High-Risk Pregnancies in the Setting of the COVID-19 Pandemic. Am J Perinatol 2020;37(8):800-08.

8. Carrascosa JM, Pastor-Nieto MA, Ruiz-González I, et al. Patch Testing During the COVID-19 Pandemic: Recommendations of the AEDV's Spanish Contact Dermatitis and Skin Allergy Research Group (GEIDAC) Recomendaciones del Grupo Español de Investigación en Dermatitis de Contacto y Alergia Cutánea (GEIDAC) de la AEDV en relación con la realización de pruebas epicutáneas durante la pandemia por SARS-CoV-2 (COVID-19). Actas Dermosifiliogr 2020;111(8):650-54.

9. McIntyre M, Robinson LR, Mayo A. Practical Considerations for Implementing Virtual Care in Physical Medicine and Rehabilitation: For the Pandemic and Beyond. Am J Phys Med Rehabil 2020;99(6):464-67.

10. Pollock K, Setzen M, Svider PF. Embracing telemedicine into your otolaryngology practice amid the COVID-19 crisis: An invited commentary. Am J Otolaryngol 2020;41(3):102490.

11. Yellowlees P, Nakagawa K, Pakyurek M, Hanson A, Elder J, Kales HC. Rapid Conversion of an Outpatient Psychiatric Clinic to a
100\% Virtual Telepsychiatry Clinic in Response to COVID-19. Psychiatr Serv 2020;71(7):749-52.

12. Morais-Almeida M, Sousa CS, Barbosa MT, Aguiar R, Benito-Garcia F. Telehealth: The future is now in allergy practice. J Allergy Clin Immunol Pract 2020;8(8):2836-7.

13. Donelan K, Barreto EA, Sossong S, et al. Patient and clinician experiences with telehealth for patient follow-up care. Am J Manag Care 2019;25(1):40-4.

14. Mair F, Whitten P. Systematic review of studies of patient satisfaction with telemedicine. BMJ 2000;320(7248):1517-20.

15. Olvera-Montes N, Reyes B, Charleston-Villalobos S, et al. Detection of Respiratory Crackle Sounds via an Android Smartphone-based System. Annu Int Conf IEEE Eng Med Biol Soc 2018;2018:1620-23.

16. Kang SH, Joe B, Yoon Y, Cho GY, Shin I, Suh JW. Cardiac Auscultation Using Smartphones: Pilot Study. JMIR Mhealth Uhealth 2018;6(2):e49.

17. Duplaga M. The acceptance of e-health solutions among patients with chronic respiratory conditions. Telemed J E Health 2013;19(9):683-91.

18. Ambrosino N, Fracchia C. The role of tele-medicine in patients with respiratory diseases. Expert Rev Respir Med 2017;11(11):893-900. 